\title{
MATHEMATICAL MODELLING OF A CIRCULAR CENTRAL SOLAR RECEIVER WITH VARIABLE DIAMETER HEADER
}

\author{
${ }^{1}$ Kaustubh Kulkarni , ${ }^{2}$ Sanjay Havaldar \\ ${ }^{1}$ Research Scholar, School of Mechanical Engineering,Dr. Vishwanath Karad MIT World Peace University, Pune, \\ India -411038 \\ ${ }^{2}$ Professor, School of Mechanical Engineering, Dr. Vishwanath Karad MIT World Peace University,Pune, India -
} 411038.

\begin{abstract}
The circular central solar receiver helps in the generation of power output in a solar power plant. This paper of research encompasses mathematical development and modelling of a circular central solar receiver with variable diameter header. This modelling is utilized for analyzing the power output in a year. A desired electrical output is generated by the modeling. Methodologies were utilized for evaluating the desired parameters involved in the modelling of the entire system such as; size of the receiver, height of the tower etc. In this modelling system, solar simulator is utilized in the place of heliostat. The working fluid in the system is water. The type of receiver involved in the modelling is a circular central solar receiver. Simulation of the thermal solar receiver system is done. The solar irradiation heat flux of $3500 \mathrm{~W} / \mathrm{m}^{2}$ is applied at the input.
\end{abstract}

Keywords: Circular central solar receiver, solar simulator, header etc.

\section{Introduction}

Central solar receivers are regarded as a renewable technology for collecting the solar radiation and converting them into thermal energy of high temperature for the production of electricity and for the purpose of chemical processing. The Soviet researchers carried out an experimental analysis of the central solar receiver system in the year 1957, where an optical system encompassing 1293 mirrors were examined for producing steam at a temperature of $400^{\circ} \mathrm{C}$. The concept of central solar receiver for the solar energy concentration and collection is contemplated on the heliostat fields, which reflects the incident radiation from the Sun into a solar receiver, which is embedded the central solar tower's top position. The solar energy, which is collected in the total field, is transmitted optically. Usually the working fluid absorbs $80-95 \%$ of the gained solar energy. This absorbed energy is pumped into the receiver and the solar tower. The working fluid then gets down from the tower and then reaches the industrial powerplant and thermoelectric power plant, which requires heat energy. Concentrated solar radiation is sent into the central solar receiver. A typical central solar receiver system encompasses many heliostats (tracking mirrors), which would be positioned in the solar tower system and which would reflect the incident solar radiation. These systems have high concentration levels when compared with the linear systems. This concentrated radiation is utilized as heat energy for producing steam and converting steam into electrical energy in a powerplant or to a thermal energy, which could be utilized for storing molten salt. Central solar receiver systems are large scale systems, which are typically constructed for powering a steam cycle. The solar receiver's central position provides a universal benefit for the collection of entire energy in one place and thus helps in transportation savings. The disadvantage from the fixed position of the solar central receiver is that collection of sunlight is limited; heliostats are mounted at right angle to the beam such that the collected energy is low hen compared with the parabolic concentrator. The collector field's size must be increased for attaining maximum light concentration efficiency. The other drawbacks of this system are; high capital costs, environmental impacts and land utilization. Flat arid regions and desserts are preferred for installation of central solar receiver systems, where plenty of solar energy is got with low land costs and endeavors.

The proposed system is a central solar receiver system, encompassing a solar tower with a solar receiver, solar simulators, which are embedded in the system in the place of heliostats, power block and a thermal energy storage system. The proposed paper revolves around the 
mathematical modeling of this central solar receiver system having header with a variable diameter. The modelling is performed with the utilization of some specified parameters. The initial part of the paper encompasses about the theoretical background of the study; then about the related works on the central solar receiver systems; mathematical modeling of the proposed system with some specifications and finally about the results of the mathematical modelling of the proposed system.

\section{Related Works}

(Basil H. Ali, S.I. Gilani, and Hussain H. Al-Kayeim 2016) carried out the mathematical modelling of a central solar receiver, which is developed on the basis of evacuated solar tubes. In their study, they designed a new receiver model, which was contemplated on the proposed evacuated solar tube and the investigation on the developed receiver's dynamic characteristics. For evaluating and optimizing the dynamic characteristics of their system, investigation was carried out on their heat conversion process of solar radiation within the receiver and where the mass and energy conversion equation are utilized for evaluating the state of the receiver components and the working fluid temperature with the analysis and calculation of the thermal losses in the receiver. The results indicated that with the proper arrangement of the tubes, with proper configuration, the plant operates with the needed pumping power. The study recommends for further analysis on friction losses of the hydraulic fluid within the tubes.

(Luca Cioccolanti, Roberto Tascioni and Alessia Arteconi 2018) conducted a mathematical modelling of the performance evaluation and operation modes creative small scale solar organic and rankine cycle plant. In the study a solar organic rankine cycle plant is embedded with a storage tank made of phase change material along with heat pipes, which are reversible, are examined with a simulation analysis. The modelling was performed utilizing TNRSYS software. The examination of the performance of the system was carried out with the given control strategy. For a period of one year, various operating modes in the system were examined. The plant gave full efficiency based on the given design parameters. The results of the simulation offered ways for frequent real plant testing.

(Sandhya Jadhav and V. Venkatraj 2018) conducted a mathematical modelling on the thermal losses of a solar central receiver in a thermal power plant. Simulation and mathematical modelling of a solar central receiver power plant embedded with a cavity receiver was done for evaluating the cavity receiver's thermal power losses. Results of the evaluation are done with the experimental results of the solar experiments. The efficiency of the receivers in the transfer and absorption of the solar energy into the working fluid was crucial in the central solar receiver as the produced energy cost, capital cost and performance of the plant had an impact by the efficiency of the receiver. Power generation results were obtained with hourly simulation. The study opts for gaining knowledge on the power plant performance and construction of the central solar receiver.

(Lachhab et.al 2019) carried out a mathematical modelling and theoretical analysis of a solar cogeneration system situated in Morocco. This mathematical modelling is based on solar cogeneration's new technology utilizing the photovoltaic cell and parabolic trough concentrator. The eminent objective of their study was to examine the parabolic concentrator's thermal performance. Matlab was utilized for simulation of the modelling. Encouraging results were obtained with the experimental analysis and finally satisfactory performance of the solar collector was obtained.

(Moukhtar et. al 2018) performed the regulation of electric power and modelling of a power plant with a central tower receiver, which is contemplated on the technique of artificial neural network. The modelling of the system was performed utilizing the artificial neural network technique. The system encompassed one hidden layer, one output parameter and two input parameters. The heat transfer fluid's mass flow rate was the output. The system's performance was analyzed experimentally and the results were referred with the system advisor model. Compatible results were observed between the outputs of the system and the adopted ANN model. Less input data and simplicity made it an appropriate model for the examination of the study. The drawback of the ANN model is that it works only with the information that is numerical and is entirely contemplated on the ability of the users.

\section{Mathematical Modelling of Circular Central Solar Receiver}

Mathematical modelling is performed for the solar central receiver system. Codes were developed for the simulation and development of the receiver. The solar energy is got by 
the solar central receiver by their reflection by the solar simulators. Then the receiver's thermal losses were examined. The solar simulators were dimensioned for receiving high temperature having high flux densities. The calculation of the below mentioned parameters are eminent aspects of the modelling of the proposed system.

\section{Equivalent Capacity}

For evaluating the amount of solar energy incident on the central circular solar receiver system $\left(Q_{\text {rec }}\right)$, the system's thermal energy capacity and rated capacity are needed. The solar receiver's equivalent capacity is the sum of the above capacities. The equivalent capacity is calculated as follows;

$$
\text { Equivalent capacity }=(\mathrm{h}+\mathrm{x}) / \mathrm{h} * \text { rated capacity }
$$

Here, h-> hours of thermal energy storage capacity.

The solar simulator system's efficiency is calculated as follows;

$\eta_{\text {total }}=$ sum of efficiencies of the components of the system

\section{Determination of reflecting area of the solar simulator}

For calculating the reflecting area of the solar simulator, $\left(\mathrm{A}_{\text {ref }}\right)$ the needed solar energy must be reflected by the solar simulator field to the central solar receiver. The reflecting area of the solar simulator is calculated as follows;

$$
\mathrm{A}_{\mathrm{s}}=\mathrm{Q}_{\text {rec }} / \mathrm{DNI}
$$

Where $Q_{\text {rec }}$ is the solar energy incident on the receiver and DNI is the incident solar radiation per unit aperture area $\left(3500 \mathrm{~W} / \mathrm{m}^{2}\right)$ for this system

\section{Thermal energy of the receiver}

The thermal solar energy incident on the receiver

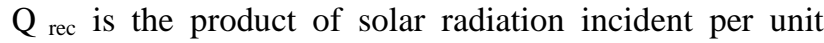
aperture area, area of the solar simulator and efficiency of the solar simulator, which is mathematically indicated as,

$$
\mathrm{Q}_{\mathrm{rec}}=\mathrm{DNI} * \mathrm{~A}_{\mathrm{s}} * \eta_{\mathrm{s}}
$$

Where, $\mathrm{A}_{\mathrm{s}^{-}}>$area of the solar simulator and $\eta_{\mathrm{s}^{-}}>$ efficiency of the solar simulator

Mean temperature of the solar simulator
The mean temperature of the solar simulator field is calculated as the sum of half of inlet and outlet temperatures of the working fluid, which is water in the proposed system. This could be mathematically indicated as;

$$
\mathrm{T}_{\text {mean }}=\left(\mathrm{T}_{\text {in, } w}+\mathrm{T}_{\text {out, }}\right) / 2=(80+30) / 2=55^{\circ} \mathrm{C}
$$

Where, $\mathrm{T}_{\text {mean }}$ is the mean temperature of the solar simulator field; $\mathrm{T}_{\mathrm{in}, \mathrm{w}}$ is the inlet temperature of the working fluid and $\mathrm{T}_{\text {out, } \mathrm{w}}$ is the outlet temperature of the working fluid.

\section{Allowable heat flux}

The allowable heat flux is calculated as the product of maximum heat flux and average peak to heat flux ratio.

In addition to the above-mentioned parameters, the thermal energy losses of the receiver such as; heat losses, coefficient of heat transfer, heat losses during emission, reflection and conduction by the solar simulator and the cost of conversion are calculated.

\section{Total radiative Power}

Classical radiative transfer theory and laws of geometrical optics are utilized by the solar concentrating systems' radiative transfer models that are macroscopic. These are contemplated on the basis of radiative transfer equation (RTE). A solar central receiver (SCR) system model was utilized for calculating the radiative transfer concept and the pertinent optics. The incident solar radiation and spectral intensity from $\hat{\mathrm{s}}$ direction on $\mathrm{dA}$ arbitrary surface element with position $\vec{r}$ and normal $\hat{n}$ is indicated as follows,

$$
\begin{aligned}
& \mathrm{I}_{\text {solar, } \lambda}(\lambda, \vec{r}, \mathrm{t}, \hat{s})=\mathrm{dQ} \text { solar }(\lambda, \vec{r}, \mathrm{t}, \hat{s}) /|\hat{s} . \widehat{n}| . \mathrm{d} \lambda . \mathrm{dA} . \mathrm{d} \Omega \text {. } \\
& \mathrm{dt} \text {. } \\
& \mathrm{Q}_{\text {solar, } \lambda}(\lambda, \vec{r}, \mathrm{t}, \hat{s})=\int_{0}^{2 \pi} \quad I_{\text {solar, } \lambda}(\lambda, \vec{r}, \mathrm{t}, \hat{s}) \mid \hat{s} . \hat{n} / . \\
& \mathrm{d} \Omega \ldots \ldots \ldots(2)
\end{aligned}
$$

Where dQ solar $(\lambda, \vec{r}, \mathrm{t}, \hat{s})$ and dQ' solar $(\lambda, \vec{r}, \mathrm{t}, \hat{s})$ are the interceptive radiative power and solar radioactive energy. This interceptive solar radiative power is intercepted along the change in area $\mathrm{dA}$, in the $\mathrm{d} \lambda$ wavelength interval and will be $\lambda$ wavelength. The incident solar radiation on the surface element $\mathrm{dA}$ of the solar simulator having a direction of reflection $\widehat{s r}$ and the normal $\hat{n}$ are the eminent parameters here. The essential fundamental property in the geometric optics that defines the reflection process within 
the thermodynamic condition of equilibrium is referred to as spectral bidirectional reflection function; which is indicated as $\rho_{\lambda}{ }^{\prime}(\vec{r}, \widehat{s r}, \widehat{s l}, \lambda)$. The mathematical indication of this function is given as follows;

$\rho_{\lambda} ",(\vec{r}, \widehat{s r}, \widehat{s l}, \lambda)=\mathrm{dI}_{\lambda}(\vec{r}, \widehat{s r}, \widehat{s l}, \lambda) / \mathrm{I}_{\lambda}(\vec{r}, \lambda, \widehat{s l}) \cdot|\widehat{s l} . \hat{n}|$. $\mathrm{d} \Omega_{\mathrm{i}}$

The reflected intensity's angular spread and less spectral attenuation are a reflecting surface's eminent spectral characteristics, which helps in maximizing the total radiative power, which is intercepted by a receiver area of $\mathrm{A}_{\text {rec. }}$

$$
\mathrm{Q}_{\text {rec }}(\vec{r})=\iiint \mathrm{I}_{\lambda, \text { rec }}(\hat{s}, \lambda, \vec{r})|\hat{s} \cdot \hat{n}| \mathrm{d} \lambda \cdot \mathrm{dA} \cdot \mathrm{d} \Omega
$$

\subsection{Metrics of performance}

The performance parameters of solar thermal concentrating systems could be classified as thermodynamic and optical systems. The metrics of optical performance are the solar concentration ratio and the optical efficiency. The parameters of thermodynamic results are the thermal efficiency of the receiver, thermodynamic conversion efficiency, which is ideal and the absorption efficiency.

\section{a. Optical efficiency}

The ratio of the radiative power of the solar receiver to the area of aperture $\mathrm{A}_{\text {rec }}$ with the maximum energy gained by the solar simulator is termed as optical efficiency, which is given by,

$$
\eta_{\text {opt }}=\int \quad Q^{\prime} \text { rec.dt/Am G. } \Delta t
$$

The total optical efficiency encompasses the atmospheric losses of attenuation, reflection, spillage, blocking, shading and cosine losses.

\section{b. Concentration ratio}

The concentration ratio is defined as the ratio of the aperture area of the concentrator to the aperture area of the receiver, which is given by,

$$
\mathrm{C}_{\mathrm{a}}=\mathrm{A}_{\mathrm{c}} / \mathrm{A}_{\mathrm{rec}}
$$

Where, $\mathrm{A}_{\mathrm{c}}$ is the aperture area of the concentrator, $\mathrm{C}_{\mathrm{a}}$ is the concentration ratio and $A_{\text {rec }}$ is the aperture area of the receiver. The energetic concentration ratio or the average area flux is given by,

$$
\mathrm{C}_{\mathrm{f}}=\mathrm{Q}^{\prime} \text { red } / \mathrm{G} \cdot \mathrm{A}_{\mathrm{rec}}
$$

Where, $\mathrm{G}$ is the normal direct irradiance.

The following shows the block diagram of the proposed system, where the mathematical modelling is to be applied.

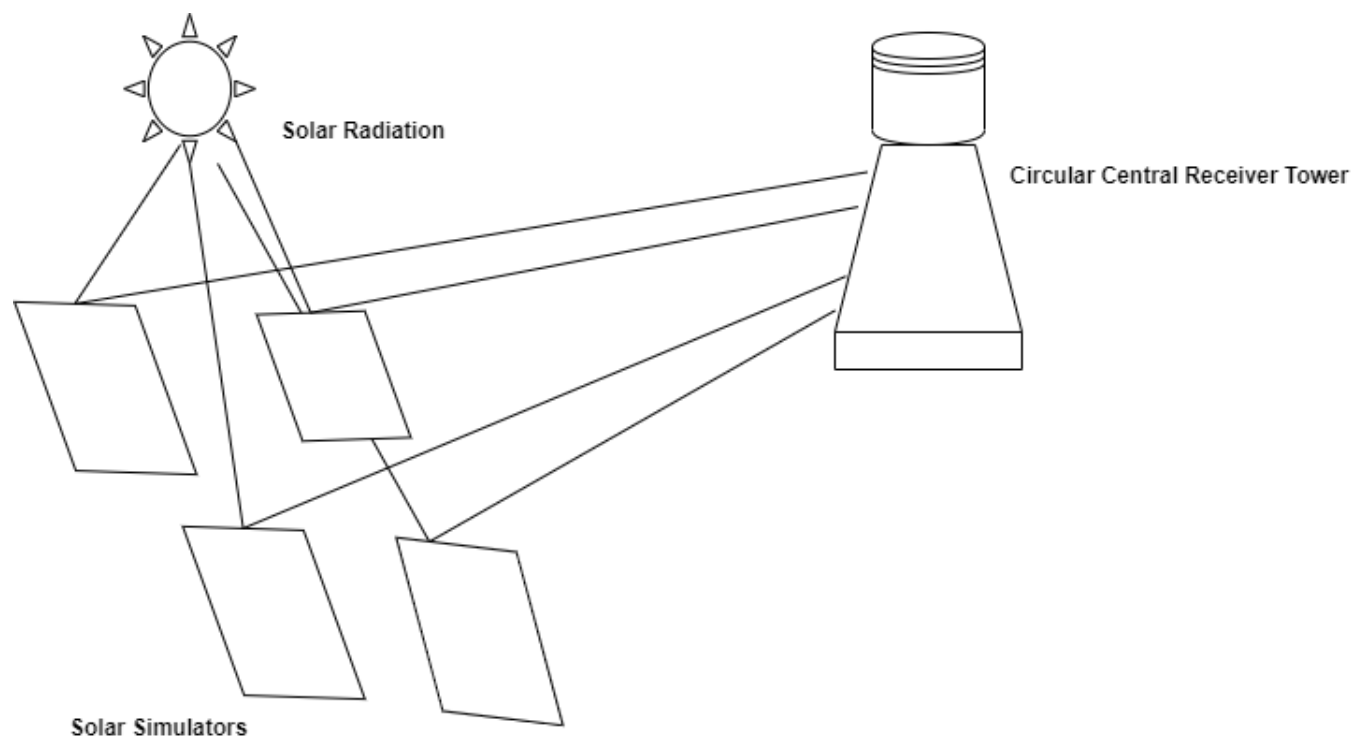

Figure 1

Block diagram used for the mathematical modelling of the circular central solar receiver
The efficiency of the receiver is assumed as equal to the entire rated capacity of the solar central receiver system. 


\section{Results and Discussion}

The design parameters for the mathematical modelling of the proposed circular central solar receiver system are given in the following table. In this system, solar simulators are utilized instead of heliostats encompassed in an ordinary central solar receiver system.

\begin{tabular}{|c|c|c|}
\hline Sl. No. & Parameters & Dimensions \\
\hline 1. & $\begin{array}{c}\text { Diameter of the bottom header inlet } \\
\text { and outlet }\end{array}$ & $50 \mathrm{~mm}$ and $15 \mathrm{~mm}$ \\
\hline 2. & $\begin{array}{c}\text { Diameter of the upper header inlet } \\
\text { and outlet }\end{array}$ & $15 \mathrm{~mm}$ and $50 \mathrm{~mm}$ \\
\hline 3. & Area of the receiver & $0.6 \mathrm{~m}^{2}$ \\
\hline 4. & Number of tubes & $50 \mathrm{~mm}$ \\
\hline 5. & Diameter of central tube & $1 \mathrm{~mm}$ \\
\hline 6. & Tube thickness & $0.1 \mathrm{LPM}$ \\
\hline 7. & Mass flow rate & $3500 \mathrm{~W} / \mathrm{m}^{2}$ \\
\hline 8. & Heat flux & $3-4$ \\
\hline 9. & Metal halide lamps (solar simulator) & Copper receiver \\
\hline 10. & Material of receiver and frame & \\
\hline
\end{tabular}

Table 1: Design parameters of the modelling of the system

The incident solar radiation was measured and recorded during a day, which has a clear sunshine. The measurement was made until mid-afternoon and reduces slowly post noon. During that time, the highest solar incident radiation was $3500 \mathrm{~W} / \mathrm{m}^{2}$ at 12 am with the highest temperature. An increase in energy and temperature was observed with an increase in solar radiation. Heat loss increases within elevation in the speed of the wind, the water flow rate is less such that heat is transferred to water and rapidly increases and is converted to steam with a rise in transfer of heat. The rise in temperature results in speed of heat transfer with an elevating efficiency of the receiver. The thermal solar energy quantity, which was shifted from the solar concentrator to the solar central receiver provides full efficiency in operation. This efficiency minimizes with lowering of time. A drop in temperature was observed during loss in energy. Thus, temperature energy losses are directly proportional. The following shows the results of wind speed versus heat losses during the analysis of the system.

Figure 2

The operation's suitability is indicated in the following graph, which is the graph between the temperature and instantaneous efficiency. 


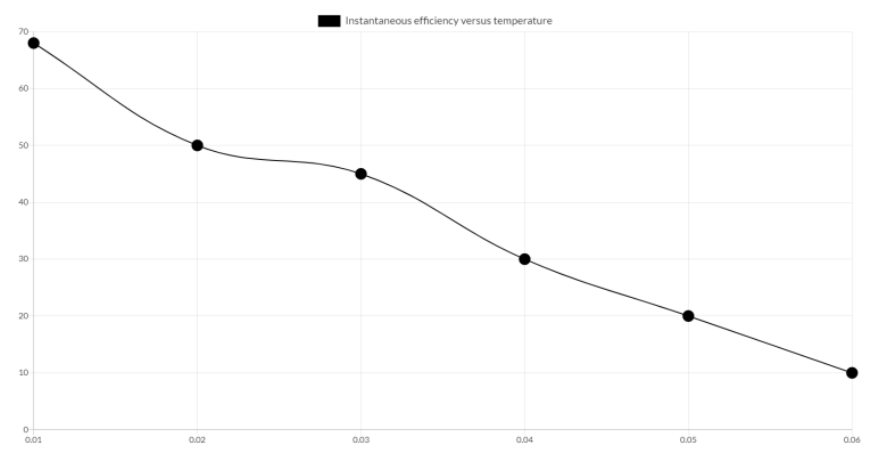

Figure 3

Instantaneous efficiency versus temperature

The variable diameter designed with the design parameters encompassing the diameters of the inlet and outlet of the header in the circular central solar receiver is shown below. The incident solar irradiance falls on the solar simulators; this beam is reflected by the simulators to the receivers, which is intercepted at a certain angle with the solar simulators. The receiver is circular with a variable diameter header, where there is a upper header and a lower header in the receiver. The mathematical modelling is performed with the above specified parameters and the simulation result is obtained for the circular central solar receiver. Matlab is utilized for obtaining the simulation results

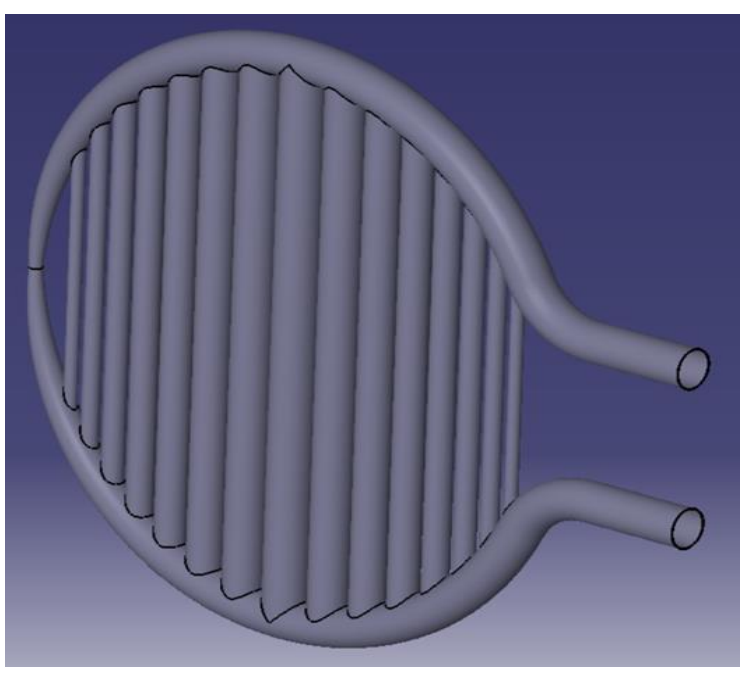

Figure 4

Simulation result of a circular central solar receiver system

A circumferential aperture is possessed by the receiver for matching the surrounding field. Here, the acceptance angles are wide. The results of modelling of the solar central receiver indicate that the thermal efficiency of the receiver varies with the surface temperature and are sensitive in nature. The receiver's thermal state remains stable chemically and the temperature of the solar central receiver is controlled by the working fluid. The temperatures in the surface of the absorbing area and the thermal losses in the solar panel area remain stable in accordance with the heat flux. Losses are observed in areas, where the solar incident energy is not absorbed. These losses vary with the level of heat flux. The examined rate of flow of water and the solar power absorbed are checked with global values and the measured data was examined mathematically. 


\section{Conclusion}

Thus, in this study, we have performed the mathematical modelling of the circular central solar receiver system possessing a header in the top and bottom having a variable diameter. The specific configuration of the system was modeled with the eminent components such as solar simulators, simulators' field, circular central solar receiver and concentrator. Mathematical modelling of the central solar receiver system is an accurate and efficient method for the optimization and design of the system without any associated costs related to the fabrication of the prototype systems. Many tools are available for the solar engineers for minimizing the construction time of the central solar receiver system. The thermal storage system assists the solar central receiver to operate during cloudy weather conditions. The efficiency of operation of the system is high during summer months. The results of the mathematical modelling of the system are compared with Matlab simulations and encouraging results were obtained. Furthermore, some of the system's configuration needs new development, extension or advanced tools for allowing high simulation flexibility and accuracy, suitably in optical problems. Thus, capital costs of the system could be minimized by a better optical design. The modelling is encouraged to encompass a control system for altering the mass flow rate of the heat transfer fluid or the number of solar simulators, which contemplates on the solar incident energy during period of time so that the working fluid do not rise above the maximum temperature of working.

\section{References}

1. "Modelling of a Central Tower Receiver Power Plant." Springerprofessional.De, https://www.springerprofessional.de/en/modellin g-of-a-central-tower-receiver-powerplant/18585760. Accessed 10 Mar. 2021.

2. "Mathematical Modelling of Operation Modes and Performance Evaluation of an Innovative Small-Scale Concentrated Solar Organic Rankine Cycle Plant." Applied Energy, vol. 221, July 2018, pp. 464-76. www.sciencedirect.com, doi:10.1016/j.apenergy.2018.03.189.

3. Basil. H. Ali, S. I. Gilani , and Hussain H. AlKayiem . Mathematical Modeling of a Developed Central Receiver Based on Evacuated Solar Tubes. EDP Sciences, 2016,doi:10.1051/matecconf/20163802005.
4. Sandhya Jadhav, V.Venkatraj. Thermal Losses in Central Receiver Solar Thermal Power Plant. 2018, doi:10.1088/1757-899X/377/1/012008.

5. Lachhab, Saad Eddin, et al. "Theoretical Analysis and Mathematical Modeling of a Solar Cogeneration System in Morocco." AIMS Energy, vol. 7, no. 6, 2019, pp. 74359. www.aimspress.com, doi:10.3934/energy.2019.6.743.

6. Robert Flesch. Daniel Maldonado Quinto. Peter Schwarzbözl . Dynamic Modelling of Molten Salt Central Receiver Systems. 2018, https://www.argesim.org/fileadmin/user_upload argesim/ARGESIM_Publications_OA/MATHM OD_Publications_OA/MATHMOD_2018_AR55 /articles/a55231.arep.55.pdf.

7. Moukhtar, Ibrahim, et al. "Electric Power Regulation and Modeling of a Central Tower Receiver Power Plant Based on Artificial Neural Network Technique." Journal of Renewable and Sustainable Energy, vol. 10, no. 4, July 2018, p. 043706. aip.scitation.org doi:10.1063/1.5029898.

8. Li, Lifeng, et al. "Optics of Solar Central Receiver Systems: A Review.” Optics Express, vol. 24, no. 14, July 2016, pp. A9851007. www.osapublishing.org, doi:10.1364/OE.24.00A985.

9. Zhu, Guangdong, and Cara Libby. Review and Future Perspective of Central Receiver Design and Performance. 2017, p. 030052. DOI.org (Crossref), doi:10.1063/1.4984395.

10. Maria Hallberg and Elin Hallme. Introducing a Central Receiver System for Industrial HighTemperature Process Heat Applications. KTH Royal Institute of Technology, 2018, https://www.divaportal.org/smash/get/diva2:1303784/FULLTEX T01.pdf.

11. Avila-Marin, A. L., et al. "Modelling Strategies for Porous Structures as Solar Receivers in Central Receiver Systems: A Review." Renewable and Sustainable Energy Reviews, vol. 111, Sept. 2019, pp. 15-33. Science Direct, doi:10.1016/j.rser.2019.03.059.

12. Yebra, L. J., et al. "Modelling and Simulation of Central Receiver Solar Thermal Power Plants." Proceedings of the 44th IEEE Conference on Decision and Control, 2005, pp. 
7410-15. IEEE Xplore, doi:10.1109/CDC.2005.1583357.

13. María de los Reyes Rodríguez Sánchez . On the Design of Solar External Receivers . Sept. 2015, https://core.ac.uk/download/pdf/44309378.pdf.

14. Alexopoulos, Spiros, and Bernhard Hoffschmidt. "Concentrating Receiver Systems (Solar Power Tower)." Solar Energy, edited by Christoph Richter et al., Springer, 2013, pp. 29-71. Springer Link, doi:10.1007/978-1-4614-5806-7_677.

15. Kreith, Frank, and Richard T. Meyer. "LargeScale Use of Solar Energy with Central Receivers: Systems Using Mirros to Direct Sunlight at a Central Receiver Can Produce Heat for Industry and Electric Utilities." American Scientist, vol. 71, no. 6, 1983, pp. 598-605.

16. Marugán-Cruz, C., et al. "District Cooling Using Central Tower Power Plant." Energy Procedia, vol. 49, Jan. 2014, pp. 1800-09. ScienceDirect, doi:10.1016/j.egypro.2014.03.191. 